\title{
System Designed for Shock and Vibration Protection Using Dry Friction
}

\author{
Yu. Kyrychuk \\ Faculty of Instrument Design and Engineering, National Technical University of Ukraine "Kyiv Polytechnic Institute", \\ Kiev, 03056, Ukraine \\ *Corresponding Author: kirichuky@email.ua
}

Copyright (C 2014 Horizon Research Publishing All rights reserved.

\begin{abstract}
The article is devoted to the analysis of a single-axle system designed for shock and vibration protection using dry friction that limits maximum acceleration of an insulated body at single high-level shocks and vibrations without a static dead band near a balance point to a specified level.
\end{abstract}

Keywords Shock Protection, Vibration Protection, Damper, Dry Friction

\section{Introduction}

Vibration protection systems with dry friction dampers are widely used in modern machine building, instrumentation engineering, aircraft and space industry $[1,2]$. In the time of high velocities and accelerations that affect instruments, there is a need to simultaneously protect instruments from vibrations and shock loads of a high amplitude.

\subsection{Recent Research and Analysis of Work}

The main advantage of dry friction dampers over rubber elastic damper vibration insulators consists in the wider range of operating temperatures. Dry friction vibration protection systems feature the unlimited resonance oscillation amplitude while the relative movement of friction surfaces is present. Therefore, a chosen dry friction force should provide a "closing" vibration insulator with resonance frequency, i.e. relative motion should be zero. Another characteristic of such systems is a dead band, i.e. uncertainty of a static balance point location within a certain narrow range of motion whose restoring force is less than a dry friction force. This entails undesirable, sometimes even inadmissible static angular misalignments of a vibration insulating body. In these cases, dry friction forces must be absent near the balance point. In spite of weighty arguments about shock and vibration protection systems [2-8], little attention has been paid to dry friction vibration insulation.

Mechanical oscillations and vibrations are induced by external forces to move a mechanical system. These vibrations impact on operation of the system and often undermine its characteristics, such as precision, efficiency and service life, as well as increase heating of parts and derate their strength, affect operator's health. If balancing of separate parts and the system as a whole is not worthwhile, various methods of vibration control are used to curb vibration influence. On the one hand, measures are taken at a design stage to reduce vibration of a machine (balancing mechanisms), on the other hand, aids are provided for protection of a machine from external vibrations (this machine from its environment) and protection of the environment and operators from machine's vibrations.

Hence, the purpose of this article is to analyze the single-axle system of vibration protection using dry friction in consideration of the following requirements:

- Maximum acceleration of an insulated body at high amplitude single shocks should be limited to a specified level;

- Maximum vibration acceleration of the insulated body should be limited to a specified level;

- There must be no static dead zone near a static point.

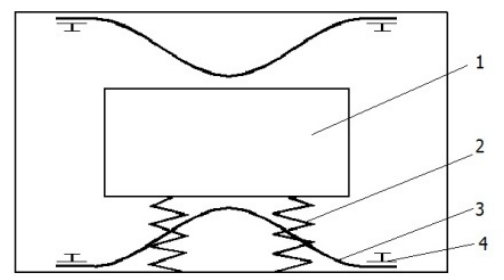

Figure1. Shock and vibration protection system with dry friction limiters

\section{Materials and Methods}

The shock and vibration protection system using dry friction limiters is shown in Figure 1 [9]. The protected body 1 is mounted on springs 2 . Limiters (dampers) comprise flat springs 3 whose ends slide in fixed bearings 4 . A friction regulation force in their location is chosen so that the body was "closed" at vibration, i.e. relative motion was absent 
with resonance frequency. When a shock occurs, it flattens a corresponding spring by the effect of the displaced body, thus enabling its relative displacement. An analytic model is shown in Figure 2.

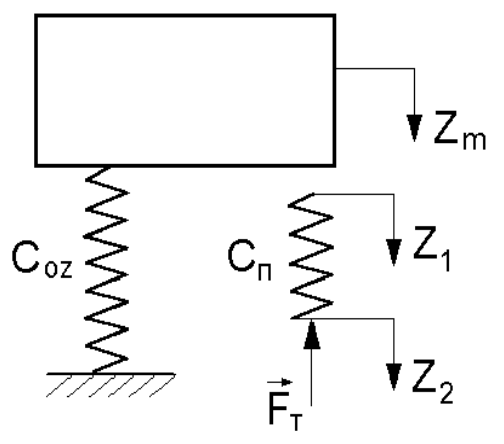

Figure 2. Analytic model of vibration protection system

Consider efficiency of this suspension as a shock protection system. Suppose a natural suspension oscillations period $T$ is much longer than shock duration $\tau$. In this case the shock can be considered as a short one, and its effect is limited to giving initial speed to the body.

$$
V_{o}=\int_{0}^{\tau} W(t) d t
$$

The principle of energy will be applied to determine maximum relative displacement and maximum absolute acceleration during the shock. Equating initial kinetic energy of the body with elastic forces and the dry friction force at work permits the formulation of the following expression:

$$
\frac{m V_{o}^{2}}{2}=A_{1}+A_{2}+A_{3},
$$

where $A_{1}=\left(C_{o z} Z_{m}^{2}\right) / 2$ is the work of elastic forces of the springs used as vibration insulators; $A_{2}=F_{T} z_{2}$ is the work of the dry friction force of flat spring ends; $A_{3}$ is the work of internal forces of flat springs; $Z_{m}$ is maximum body displacement; $z_{2}$ is the motion of flat spring ends; $m$ is the mass of the body.

Proceed to expression for $A_{3}$. This expression is given by:

$$
A_{3}=\int_{0}^{Z_{1}} P_{d}\left(z_{1}\right) d z_{1},
$$

where $P_{d}\left(z_{1}\right)$ is dependence of $P_{d}$ force influencing the flat spring on its deflection $z_{1}$. This force arises while the insulated body moves.

The following relationship exists:

$$
z_{1}=-l\left(\xi^{\prime \prime} / \sqrt{\frac{P_{d} l}{C_{n}}}\right),
$$

where $l$ is the length of a flat spring; $C_{n}$ is stiffness of the limiter (spring); $\xi^{\prime \prime}$ is the numerical value of coordinates of any point of a cycle curve that is dependent on the form of a flat spring and has been taken from tables and diagrams of elastic parameters arranged for ease of calculations using the method of elastic parameters [2].

The equation (3) can be expressed as

$$
P_{d}=-\left((\xi ")^{2} l C_{n} / z_{1}^{2}\right) .
$$

Inserting expression (3) into expression (2), we shall get the following:

$$
A_{3}=\int_{0}^{Z_{1}} \frac{\left(\xi^{\prime \prime}\right)^{2} l C_{n}}{z_{1}^{2}} d z_{1} .
$$

A choice of parameter $\xi^{\prime \prime}$ [2] is worthy of special mention. It depends on the shape of a spring, more exactly on the spring accent angle. This parameter can be expressed as a linear function of $\xi^{\prime \prime}\left(z_{1}\right)$ to simplify calculations:

$$
\xi^{\prime \prime}\left(z_{1}\right)=0.05964 z_{1}-0.3671
$$

Inserting this expression into expression (5), we shall get the following:

$$
A_{3}=C_{n} l\left(0,00357 z_{1}-0,13476\left(1 / z_{1}\right)\right) .
$$

The flat spring ends displacement $z_{2}$ can be expressed in terms of its deflection $z_{1}$ :

$$
z_{2}=L-\Delta l\left(z_{1}\right)
$$

where $L$ is a distance between flat spring bearings; $\Delta l\left(z_{1}\right)=\eta^{\prime \prime} / \xi^{\prime \prime}\left(z_{1}-H\right)$ is the function of length change $l$ of the flat spring subject to deflection change $z_{1} ; H$ is the height of the unloaded spring; $\eta^{\prime \prime}=1-0.082 z_{1}$ is the numerical value of coordinates of any point of the cycle curve that is dependent on the form of a flat spring and has been taken from tables and diagrams of elastic parameters.

Thus, we have:

$$
z_{2}=L-\frac{1-0.082 z_{1}}{0.05964 z_{1}-0.3671}\left(z_{1}-H\right) .
$$

Inserting the values of $A_{1}, A_{2}, A_{3}$ and expression (7) into the formula (1), we shall get the following: 


$$
\begin{aligned}
& \frac{m V_{o}^{2}}{2}=\frac{C_{O Z} Z_{m}^{2}}{2}+C_{n} l\left(0,00357 z_{1}-0,13476 \frac{1}{z_{1}}\right)+ \\
& +F_{T}\left(L-\frac{1-0.082 z_{1}}{0.05964 z_{1}-0.3671}\left(z_{1}-H\right)\right)
\end{aligned}
$$

A connection between the parameters of a shock protection system and the maximum acceleration of the body is established by the formula

$$
z_{1}=Z_{m}-\Delta,
$$

where $\Delta$ is clearance between the body and a dry friction damper (flat spring), which is chosen so that dampers do not touch the body mounted on insulators in default of shocks.

$$
\text { Assuming }\left(n_{1 Z}\right)^{2}=C_{O Z} / m, \quad n_{2}^{2}=C_{n} / m \text {, }
$$
$k=F_{T} / m g$, we shall get the following:

$$
\begin{aligned}
& V_{0}^{2}=\left(n_{1 Z}\right) Z_{m}^{2}+2 n_{2}^{2} l\left(0,00357\left(Z_{m}-\Delta\right)-0,13476 \frac{1}{Z_{m}-\Delta}\right)+ \\
& +2 g k\left(L-\frac{1-0.082\left(Z_{m}-\Delta\right)}{0.05964\left(Z_{m}-\Delta\right)-0.3671}\left(\left(Z_{m}-\Delta\right)-H\right)\right)
\end{aligned}
$$

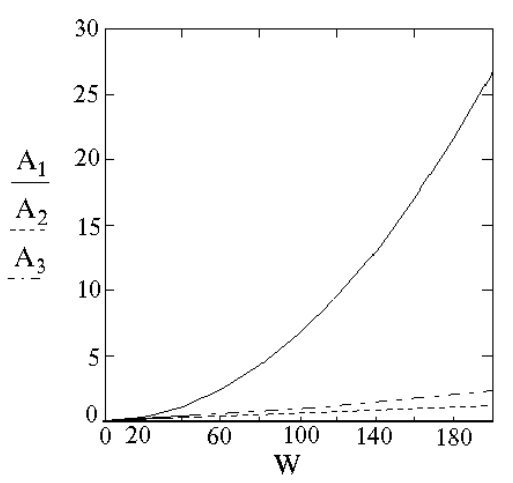

Figure 3. Dependence of the work $A_{1}, A_{2}$ and $A_{3}$ on the shock acceleration $W$ of the body

For example, Figure 3 shows dependence of the work $A_{1}$, $A_{2}$ and $A_{3}$ on the shock acceleration of the body. The plotted graph includes the following values: $C_{n}=21,3 \mathrm{~kg} / \mathrm{cm}$; $C_{O Z}=213,294 \mathrm{~kg} / \mathrm{cm} ; H=11.5 \mathrm{~mm} ; Z_{m}=5 \mathrm{~mm} ;$ $F_{T}=46 \mathrm{~N} ; W_{m}=0 . .250 \mathrm{~g} ; \Delta=0,15 \mathrm{~mm} ; l=70 \mathrm{~mm}$; and $L=67,5 \mathrm{~mm}$. The values were inserted into transformed formulae of the work $A_{1}, A_{2}$ and $A_{3}$. This figure shows that the formula (10) is true for accelerations of up to $30 \mathrm{~g}$. When acceleration is greater than $30 \mathrm{~g}$, the work $A_{2}$ and $A_{3}$, namely the effect of the dry friction force and flat spring stiffness $C_{n}$, can be ignored because of its smallness. Given that $W_{m} \approx n^{2} Z_{m}$, the natural oscillation frequency of the vibration protection system will be expressed as $n_{1 Z}=\frac{g r_{m}}{V_{O}}$, where $r_{m}=\frac{W_{m}}{g}$ is a maximum overload.

Maximum shock displacement equals $Z_{m}=\left(\left(r_{m}-k\right) g\right) / n_{1 Z}^{2}$.

Consider the effect of vibration, assuming that base displacement occurs in accordance with the law $\xi=\xi_{0} \sin \omega t$. Since vibration oscillations take place within the clearance of $\Delta$, the body motion equation can be expressed as $x^{\prime \prime}+n_{1 Z^{2}}^{2}=-W_{O C}=-\xi^{\prime \prime}$, where $x^{\prime \prime}$ is relative displacement.

A body acceleration amplitude equals

$$
W_{Z T A}=\left(W_{O C}+x^{\prime \prime}\left(=\left(\xi_{0}+a\right) \omega^{2}\right.\right.
$$

where $a=\frac{n^{2}}{n^{2}-\omega^{2}}$ is a relative displacement amplitude, $n$ is the frequency of body oscillation.

Since $a \leq \Delta$, we must make the worst of $a=\Delta$, i.e. $W_{Z T A}^{\max }=\left(\xi_{O}+\Delta\right) \omega^{2}$, assuming that interaction between the body and flat spring is absolutely inelastic at the moment of their contact without rebound. Thus, if a stopper exists, the absolute acceleration of the body will be limited even during resonance. Note that the acceleration $W_{Z T A}^{\max }$ can be represented as the equation $W_{Z T A}^{\max }=n_{1 Z}^{2}$. Qualitative dependence of body acceleration on frequency is shown in Figure 4.

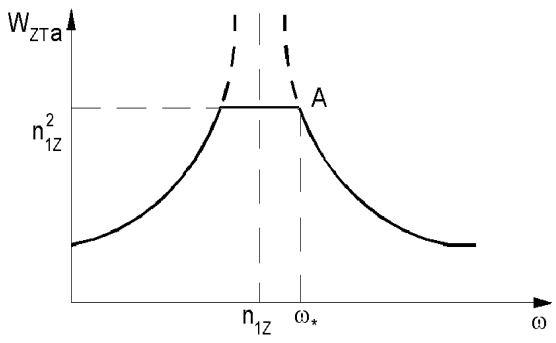

Figure 4. Dependence of the body acceleration $W$ on the frequency $\omega$

A maximum amplification ratio equals

$$
\rho=\frac{W_{Z T A}}{W_{Z A}}=1+\frac{\Delta}{\xi_{O}} .
$$

The vibration protection effect in dry friction systems takes place with frequencies that are $\sqrt{2}$ times higher than the natural oscillation frequency of the vibration protection system.

Hence, the clearance $\Delta$ should be chosen to satisfy the 
equation $\omega_{*}=\sqrt{2} n_{1 Z}$ (Fig. 4.). An increase in $\Delta$ is unadvisable because it entails an increase in the maximum vibration acceleration of the body. A decrease is also unadvisable since this leads to the increase of an interval with the constant acceleration $W_{Z T A}$ and the consequent enlargement of the area where vibration protection is not effective. Thus, assuming $\omega_{*}=\sqrt{2} n_{1 Z}$, we shall find from $W_{Z A^{*}}\left(n^{2} /\left(n_{1 Z}^{2}-\omega_{*}\right)\right)=n^{2} \Delta_{*} \quad$ that $\Delta_{*}=W_{Z A} / n_{1 Z}^{2}$, where $W_{Z A^{*}}$ is the base value of acceleration with the frequency of $\omega_{*}$.

Thus, $\Delta_{*}$ represents theoretical clearance between the body and a dry friction damper (flat spring). Assuming $w_{Z A}=1,7 g ; n_{1 Z}=47,6 \approx 48 \mathrm{~Hz} ; \omega_{*}=80 \mathrm{~Hz}$, we find $Z_{m}=0,1 \mathrm{~mm}$. Hence, oscillations actually occur within the clearance of $\Delta=0,15 \mathrm{~mm}$.

\section{Conclusions}

The above system designed for shock and vibration protection allows you to lessen shock loads by 8 times and vibration accelerations by 1.7 times. In addition, a static dead zone near a balance point is absent because the oscillation of the body occurs within $0.1 \mathrm{~mm}$ clearance.

The outlook for future development: This author finds it reasonable that the new shock and vibration protection system can be used in navigation equipment of aircraft.

\section{REFERENCES}

[1] M.Z. Kolovskii, Nonlinear theory of vibration isolation systems, Moscow, USSR: "Science", 1976.

[2] E.P. Popov, Theory and Design of flexible elastic rods, Moscow, USSR: "Science", 1986.

[3] V.B. Karpyshin, Vibrations and shocks in radio, Moscow, USSR: "Soviet radio", 1981.

[4] Alan R. Klembczyk, Introduction to Shock and Vibration Isolation and Damping Systems, IMAC-XXVII: Conference \& Exposition on Structural Dynamics, 58 - 66, 2009.

[5] Malcolm J. Crocker, Handbook of Noise and Vibration Control, John Wiley \& Sons Inc., New York NY, 2007.

[6] S. W. Yoon, S.W. Lee, N.C. Perkins, and K. Najafi, Vibration Sensitivity of MEMS Tuning Fork Gyroscopes. Proceeding of IEEE SENSORS 2007 Conference Atlanta, Georgia, USA, 115-119, 2007.

[7] Dimitry V. Balandin, Nikolai N. Bolotnik, Pilkey D. Walter, Optimal Protection from Impact, Shock and Vibration, March 7, CRC Press, 2001

[8] S.V.Purtsezov, Optimal protection of two-degree-of-freedom system from shock and vibration, PHYCON '03 Proceedings of the 2003 International Conference on Physics and Control Volume 4, 1206 - 1208, 2003.

[9] A.M. Veprik, Vibration protection of critical components of electronic equipment in harsh environmental conditions, Journal of Sound and Vibration. 259 (1), 161-175, 2003.

[10] Elena N. Bezvecilna, Yurii V Kyrychuk, Systems of protection against vibration and shock, Monograph. Zhytomyr, ZhSTU, 2009. 